\title{
Evaluation of DSM Performance with Mixed DSL Services and Measured Crosstalk Channels
}

\author{
Neiva Fonseca, Darlene Neves, Ana Claudia Gomes, Márcio Conte, \\ Boris Dortschy, Klas Ericson, Jaume Rius I Riu, Evaldo Pelaes and Aldebaro Klautau
}

\begin{abstract}
In most works published so far, dynamic spectrum management (DSM) techniques are simulated assuming a single service (e.g. ADSL) and classical copper-pair channel models described in standards for spectrum management. This work presents an evaluation of DSM algorithms based on measured channels and with multiple services. A measurement campaign was performed to completely characterize scenarios by measuring the direct and far-end crosstalk transfer functions. Results for a total of two scenarios of ten twisted pairs (200 measurements), involving both equal and non-equal length cables are presented. The work also presents a thorough investigation of state-ofart DSM algorithms (namely, ISB, ASB, SCALE and IWF) simulated in multiple services (namely, ADSL, ADSL2+ and VDSL2) scenarios.
\end{abstract}

Index Terms-Digital Subscriber Lines, Power Allocation, Dynamic Spectrum Management (DSM), FEXT Crosstalk.

\section{INTRODUCTION}

One of the major impairments in digital subscriber line (DSL) networks is the crosstalk among users sharing a binder. Crosstalk arises because the individual wires in a cable of twisted pairs radiate electromagnetically. There are two basic types of crosstalk: near-end (NEXT) and far-end crosstalk (FEXT) [1]. NEXT occurs from signals traveling in opposite directions on two twisted pairs (or from a transmitter to a near-end receiver). FEXT results from signals traveling in the same direction on two twisted pairs (or from a transmitter to a far end receiver) [1]. Typically, NEXT is a stronger interferer than FEXT, because it is not attenuated by traveling the entire length of the line. NEXT, however, can be avoided with the use of frequency division duplexing (FDD) or time division duplexing (TDD) to separate upstream and downstream signals (this is done in most of the current DSL systems). If NEXT is suppressed in a binder, FEXT becomes the main crosstalk impairment, which is reinforced by the current trend toward shorter local loops. Dynamic spectrum management (DSM) is a technique to combat crosstalk that outperforms the currently adopted static spectral masks. DSM has received considerable attention in the last years [2]-[5], with reports of an increase in rate of up to $150 \%$ in comparison to current practice.

The DSM algorithms are typically evaluated through simulations that, among other simplifying assumptions, adopt empirical channel models available in standards for spectrum management [6], [7]. These models include the direct and

The authors are with the Signal Processing Laboratory, Federal University of Pará, DEEC, CEP: 66075-900, Belém-PA, Brazil and with the BAR Group, Ericsson, Kista, Sweden. E-mails: \{mneiva, dmn, acgomes, marciomm, pelaes, aldebaro\}@ufpa.br and \{boris.dortschy,klas.ericson,jaume.rius.i.riu\}@ericsson.com. crosstalk channels among the copper pairs. The crosstalk models are called worst-case because of their "conservative" design, in the sense that in $99 \%$ of the cases, the model suggests a stronger crosstalk than the one actually measured [1]. Another aspect of the DSM literature is that typically DSM is activated only for target modems of a specific service (e.g. ADSL), while modems running other services (e.g. ADSL2+, VDSL2, etc.) do not use DSM. These other modems use static power spectral densities (PSDs) instead, and are part of the alien modems set, which simply generate interference to the target modems in the simulation. This work presents simulation results that are more realistic than the ones typically presented in the literature in both aspects.

With respect to channel modeling, this work presents results of a cable measurement campaign and uses the measured channels for DSM simulations. It should be noticed that the standard models match very well the direct channels. In contrast, the crosstalk channels obtained from the standard models are of course different from the actual ones. For example, a simulation may have several pairs with exactly the same crosstalk channel, while in practice the channels are different for each copper pair.

The work also presents results for different services simultaneously running DSM. Nowadays, companies are interested on evaluating the impact of DSM on legacy systems [8] and also the coexistence of ADSL, ADSL2+ and VDSL2 in the same binder (these are the services attracting most of the commercial interest).

Also, the DSL community has strong interest on crosstalk modeling for advanced services such as MIMO (multiple input and multiple output) systems [9], [10]. Several works have been published with crosstalk measurements and comparisons with the classical (standardized) crosstalk models [11]-[13]. In this context, the measurements presented in this work constitute another contribution.

The work is organized as follows. Section II briefly introduces the DSM problem and describes the IWF [14], ISB [3] and SCALE [4] algorithms, which basically comprises the state of art of DSM algorithms. Section III regards the performed measurement campaign, including a review of the FEXT crosstalk standard model, details about the configuration setup adopted and measurements results. Section IV presents an evaluation of DSM algorithms (IWF, ISB and SCALE) performance assuming both measured and standard channels. Section $\mathrm{V}$ consists of the conclusion and suggestions for further work. 


\section{DynAmic SPectrum MANAGEMEnT FOR DSL}

\section{A. The DSM problem}

Discrete multi-tone (DMT) modulation is a modulation technique that divides the available spectra in a number of $\Delta_{f}$-spaced independent sub-channels or tones [1]. Most DSL standards adopt DMT. It is assumed in this work DMTbased DSL services, in which transmission can be modeled independently on each tone as

$$
\mathbf{y}_{k}=\mathbf{H}_{k} \mathbf{x}_{k}+\mathbf{z}_{k}
$$

where:

- $\mathbf{x}_{k}=\left[x_{k}^{1}, \ldots, x_{k}^{n}\right]^{T}$ is the vector that contains transmitted signals on tone $k$

- $\mathbf{y}_{k}=\left[y_{k}^{1}, \ldots, y_{k}^{n}\right]^{T}$ is the vector of received signals on tone $k$

- $\mathbf{z}_{k}=\left[z_{k}^{1}, \ldots, z_{k}^{n}\right]^{T}$ is the vector of additive noise (background plus alien noises) on tone $k$

- $\mathbf{H}_{k}$ is the $N \times N$ channel matrix on tone $k$, which provides information about the channel from transmitter $m$ to receiver $n$ on tone $k$ and is given by:

$$
\mathbf{H}_{k}=\left[\begin{array}{rrr}
h_{k}^{11} & \ldots & h_{k}^{1 N} \\
\vdots & \ddots & \vdots \\
h_{k}^{N 1} & \ldots & h_{k}^{N N}
\end{array}\right]
$$

where $N$ denotes the total number of users, the directchannels are the diagonal elements and the off-diagonal elements are the crosstalk channels.

The transmitted power spectrum density (PSD) is given by

$$
s_{k}^{n}=\varepsilon\left\{\left|x_{k}^{n}\right|^{2}\right\},
$$

where $\varepsilon\{$.$\} denotes expected value.$

DSM algorithms try to solve the so-called spectrum management problem (see, e.g., [15]), which can be posed as

$$
\begin{aligned}
\max & \sum_{n=1}^{N} \omega_{n} R^{n} \\
\text { such that } & \sum_{k=1}^{K} P_{k}^{n} \leqslant P_{n}^{\max }, \text { for } n=1,2, \ldots, N
\end{aligned}
$$

where:

- $K$ denotes the total number of tones used

- $R^{n}$ denotes the total bit rate of user $n$

- $P_{k}^{n}$ denotes the power allocated by user $n$ at tone $k$

- $w$ is a non-negative constant that allows one to give different priorities to the users [4]

- $P_{n}^{\max }$ is the total amount of power that user $n$ has available and is imposed by the modem analog front end.

Considering that each modem treats interference from other modems as noise, the achievable bitloading of user $n$ on tone $k$ can be written as [1]:

$$
b_{k}^{n}=\left(1+\frac{1}{\Gamma} \frac{\left|h_{k}^{n, n}\right|^{2} s_{k}^{n}}{\sum_{n \neq m}\left|h_{k}^{n, m}\right|^{2} s_{k}^{m}+\sigma_{k}^{n}}\right) .
$$

where $\sigma_{k}^{n}$ is the normalized noise power density given by

$$
\sigma_{k}^{n}=\varepsilon\left\{\left|z_{k}^{n}\right|^{2}\right\}
$$

The data rate on line $n$ is thus

$$
R^{n}=F_{s} \sum_{k} b_{k}^{n}
$$

in bits per seconds, where $F_{s}$ is the symbol rate [1].

The bitloading allows the modem to adapt to the changing line conditions by dynamically varying the constellation used in each tone. Power loading allows the modem to vary the transmitted power at each tone, which makes it possible for the modems to tune the SNR at the receiver [16].

The next subsections briefly describe the DSM algorithms used in this work.

\section{B. Iterative Water-filling Algorithm - IWF}

The IWF [14] is a power allocation algorithm and adopts the concept of a noncooperative game where each user tries to selfishly maximize its own data-rate under a given power constraint $P_{n}^{\max }$. With IWF, the PSD of each user $n$ on tone $k$ is adjusted according to

$$
s_{k}^{n}=\max \left(W-\frac{\Gamma\left(\sigma_{k}^{n}+\sum_{m \neq n} s_{k}^{m}\left|h_{k}^{m n}\right|^{2}\right)}{\left|h_{k}^{n n}\right|^{2}}, 0\right),
$$

where $W$ is the water level constant which is chosen so that the power constraint is met [14] and $\Gamma$ denotes the SNR-gap [1].

IWF iteratively tries to have each modem setting its total power $P_{n}=\sum_{k=1}^{K} P_{k}^{n}$ to the minimum possible value that still allows the modem to achieve its target data-rate [14].

\section{Iterative Spectrum Balancing Algorithm - ISB}

The ISB [3] was inspired on the optimal spectrum balancing (OSB) algorithm [2], which can find optimal solutions to the spectrum balancing rate adaptive problems, but has a complexity that increases exponentially with the number of users. ISB has a much smaller computational cost than OSB, basically because it uses a dual Lagrangian decomposition to exchange a grid-search by a line search. ISB typically achieves solutions close to the optimal, in spite of the lack of proofs of its optimality. Its implementation should be done in a centralized point of the network, the spectrum management center (SMC).

The solution of the power allocation at a given tone $k$ for user $n$ is:

$$
P_{k}^{n}=\arg \max \left(\sum_{n=1}^{N} \omega_{n} F_{s} b_{k}^{n}-\sum_{n=1}^{N} \lambda_{n} P_{k}^{n}\right)
$$

and it must be solved by a brute force search. The ISB algorithm iterates through the users, optimizing the PSD of each user in turn.

\section{Low-Complexity Distributed Algorithm - SCALE}

This algorithm is proposed in [4] and is a low complexity algorithm to solve rate adaptive problems. Its implementation could be distributed over the network, but the SMC would still be necessary. That is because, as in ISB, this algorithm also needs all the crosstalk transference functions of all users to achieve its best performance (near-optimal, very close to 
ISB). If this information is not available, SCALE degrades to IWF.

The SCALE algorithm approximates the initial objective function, Eq. (3) as a convex problem. This reduces drastically the computational cost. The update equation proposed in [4] to iteratively refine the PSDs is:

$$
P_{k}^{n}=\frac{\omega_{n} \alpha_{k}^{n}}{\lambda_{n}+\sum_{m \neq n} h_{k}^{m n} \omega_{n} \alpha_{k}^{n} \frac{S N R_{k}^{n}\left(P^{k}\right)}{h_{k}^{n n} P_{k}^{n}}},
$$

where $S N R_{k}^{n}$ is a signal-to-noise ratio and $\alpha_{k}^{n}=\frac{S N R_{k}^{n}}{1+S N R_{k}^{n}}$.

\section{Measurements of the Crosstalk Channels}

Some DSM algorithms assume full knowledge of the channel, which means that all the direct and FEXT coupling transfer functions are available for the optimization process (NEXT channels are out of focus of this work). This section initially reviews the FEXT crosstalk standards models, describes the performed measurements procedure to acquire the channel transfer functions presented, and finally provide the obtained measurements curves and commentaries.

\section{A. FEXT standard crosstalk model}

The current standard T1.417 [6] describes the "1\% worst case" model for FEXT coupling, which so far is being used in DSM simulations. The FEXT transfer function magnitude (this model disregards phase) is modeled as:

$$
F E X T[f, n, l]=|H(f)|^{2} \cdot X_{F} \cdot n^{0.6} \cdot l \cdot f^{2}
$$

where $|H(f)|$ is the insertion gain channel transfer function, $X_{F} \cdot n^{0.6}$ is a coupling constant with $X_{F}=7.74 \times 10^{-21}$, $n$ represents the number of disturbers, $l$ is the coupling path length (in feet) and $f$ the frequency $(\mathrm{Hz})$.

\section{B. Configuration setup}

The FEXT coupling transfer functions were measured in a laboratory environment, with a single test equipment and unrolled cables. The configuration setup used for FEXT transfer function measurements ${ }^{1}$ is shown in Fig. 1. The output power of the 4395A Network Analyzer was assumed as $15 \mathrm{dBm}$, and split into two paths by the $87512 A / B$ Transmission/Reflection Test Set: one path is used as a reference signal and the other one as a test signal that is applied to the DUT (twisted pair or cable under test), which means that the effective inserted signal is about $7.5 \mathrm{dBm}$. In order to assure the impedance match, the twisted pairs to be measured are connected to the instrument through 2 balums (North Hills, Wideband Transformer, 0311LB, $10 \mathrm{KHz}-60 \mathrm{MHz}, 50$ ohms UNB 100 ohms BAL), and for FEXT measurements, a 100 ohms resistor are connected to in the end of the pairs under test, as well.

\footnotetext{
${ }^{1}$ The results of the measurement campaign referred in this work also included the direct transfer function measurement of the scenarios to be used in the simulations, but these direct channel curves are conveniently omitted because they follow the current standard models as expected.
}

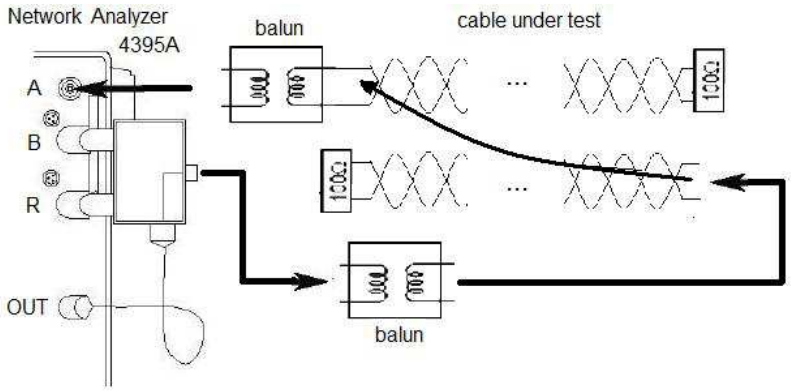

Fig. 1. FEXT transfer function measurements configuration setup.

\section{Measurements Results}

A total of 200 direct and FEXT transfer functions were measured to characterize 2 different scenarios, which the diagrams are shown in Fig. 2(a) and Fig. 2(b). Nevertheless, only the 90 FEXT coupling function regarding the scenario 2 are illustrated in this paper. In order to emphasize the differences and particularities of each FEXT-pair-to-pair coupling function, the curves were arranged in different configurations in Fig. 3, where each one contains all the pairs that theoretically should have an equal FEXT coupling transfer function response (same behavior varying in frequency). The measurements curves are identified following the legends presented in Fig. 4, according to the copper pairs distribution in the cable.

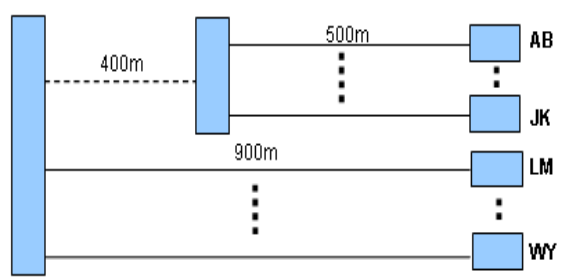

(a) Scenario 1

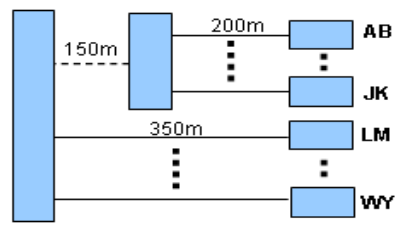

(b) Scenario 2

Fig. 2. Scenarios diagrams.

\section{Simulation Results}

This section presents simulations results that examines the performance of DSM algorithms assuming both measured and standard channels models. The simulated scenarios were composed from measurements (both direct and FEXT coupling) depicted in Fig. 2(a) and Fig. 2(b). For all simulations, was assumed ADSL, ADLS2+ and VDSL2 frequency bandplans and the ANSI VDSL Noise A as background noise [1]. Results 


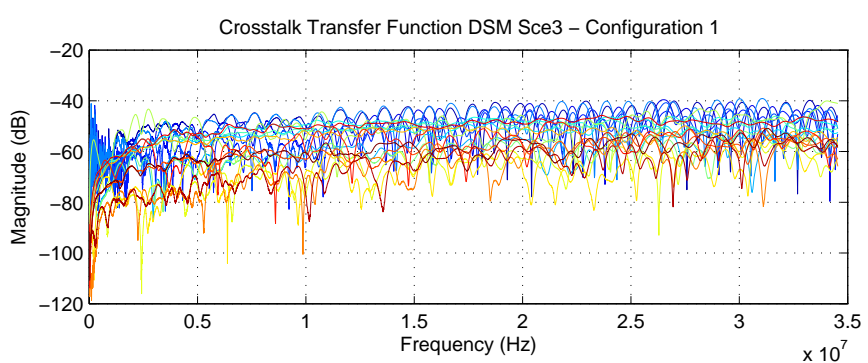

(a) Configuration 1 .

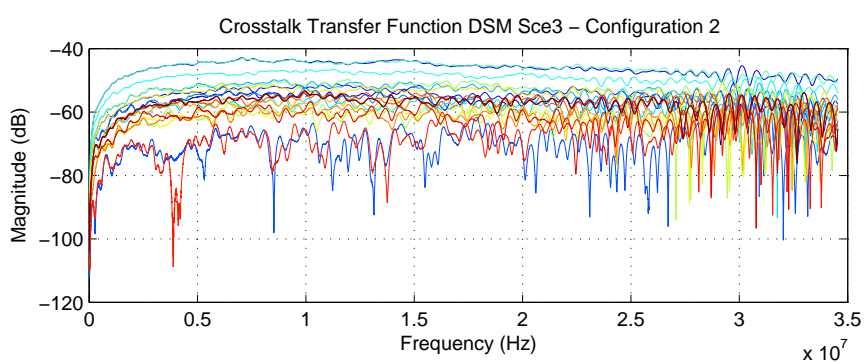

(b) Configuration 2.

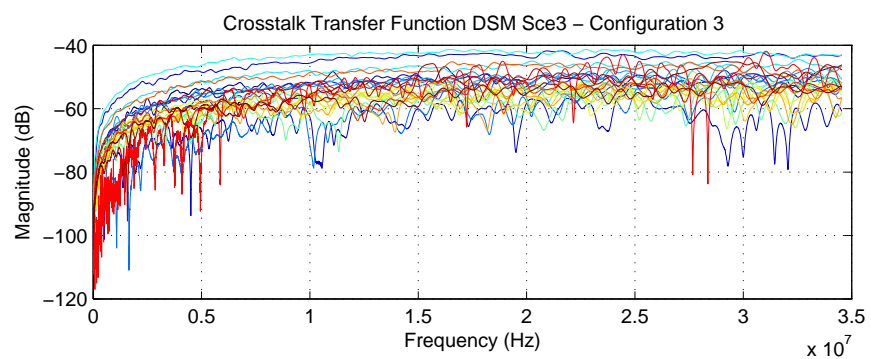

(c) Configuration 3.

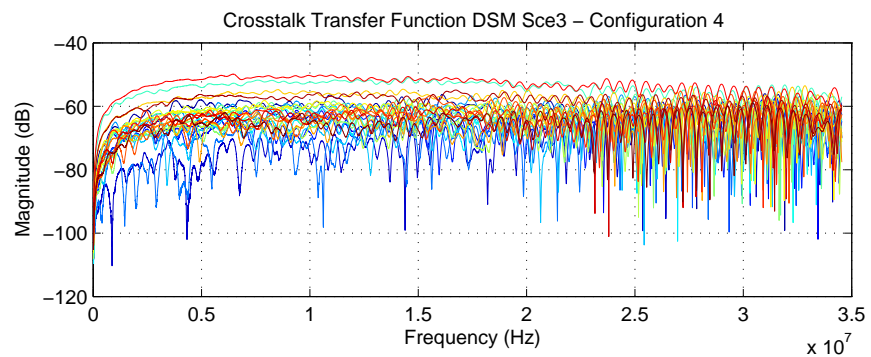

(d) Configuration 4 .

Fig. 3. Scenario 2 - Configurations.

with DSM were obtained simulating the IWF [14], ISB [3] and the SCALE [4] algorithms described in previous sections.

\section{A. Downstream transmission with multiples services and no DSM.}

For this simulation we assume the use of static spectrum management (SSM) [6], where each user maximizes its own performance without considering the performance of others lines [17]. In this case the performance is based on the levels of worst-case crosstalk interference [18].

This simulation was divided in two parts: the first simulation with five equal length users (two ADSL modems and three
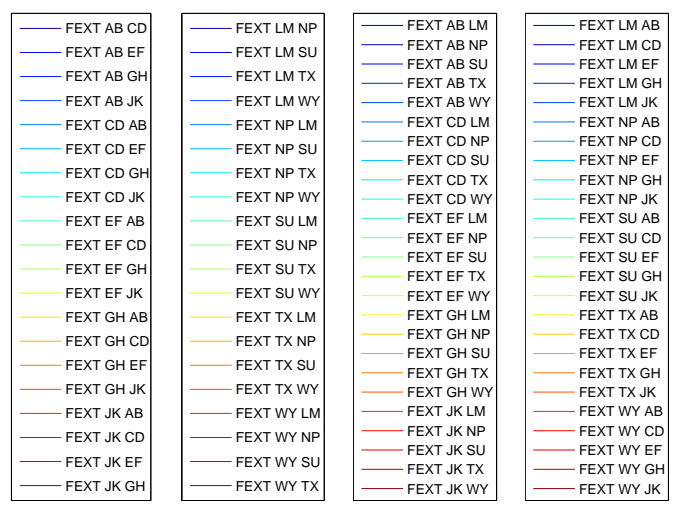

(a) Legend 1. (b) Legend 2. (c) Legend 3. (d) Legend 4.

Fig. 4. Legends of each configuration of scenario 2 .

ADSL2+ modems) direct connected at the Central Office (Fig. 5). The second simulation consists of a mixed CO/RT scenario with ten-users (three ADSL modem, six ADSL2+ modems and one VDSL modem) as depicted in Fig. 6. The goal is compare the bit rate capacity achieved when using SSM in measured and standard channels models.

Table I and Table II shows the simulations results for the five-users scenario and ten-users scenario, respectively. In both simulations the measured channel achieved bit rates higher than the simulations with standard channels models, as expected given the lower level of crosstalk compared to the standard "worst case" model.

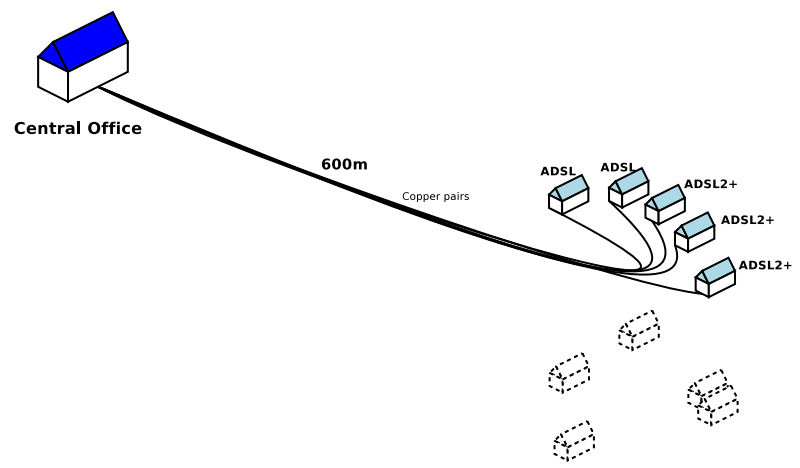

Fig. 5. Five equal length users with multiples services.

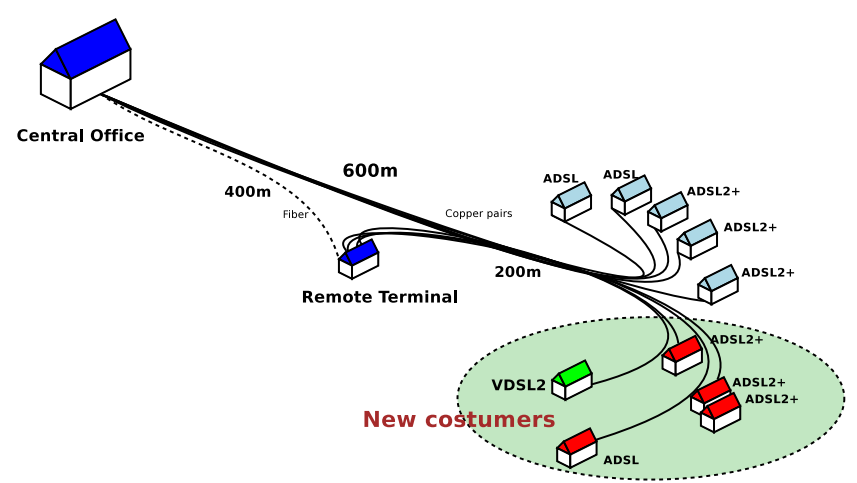

Fig. 6. Ten non-equal length users with multiples services. 
TABLE I

STANDARD DOWNSTREAM BIT RATE CAPACITY (MBPS)

\begin{tabular}{|c||c|c|c|}
\hline Lines & Service & $\begin{array}{c}\text { Calculated } \\
\text { channel }\end{array}$ & $\begin{array}{c}\text { Measured } \\
\text { channel }\end{array}$ \\
\hline \hline USR1 & ADSL & 11.10 & 12.64 \\
\hline USR2 & ADSL & 11.10 & 13.94 \\
\hline USR3 & ADSL2+ & 19.91 & 25.95 \\
\hline USR4 & ADSL2+ & 19.91 & 25.34 \\
\hline USR5 & ADSL2+ & 19.91 & 28.51 \\
\hline
\end{tabular}

TABLE II

BIT RATE CAPACITY (MBPS) IN DOWNSTREAM DIRECTION WHEN REMOTE TERMINAL CONNECTED USERS ARE INCLUDED

\begin{tabular}{|c||c|c|c|}
\hline Lines & Service & $\begin{array}{c}\text { Calculated } \\
\text { channel }\end{array}$ & $\begin{array}{c}\text { Measured } \\
\text { channel }\end{array}$ \\
\hline \hline USR1 & ADSL & 10.16 & 12.62 \\
\hline USR2 & ADSL & 10.16 & 13.82 \\
\hline USR3 & ADSL2+ & 16.41 & 24.18 \\
\hline USR4 & ADSL2+ & 16.41 & 24.87 \\
\hline USR5 & ADSL2+ & 16.41 & 25.52 \\
\hline USR6 & VDSL2 & 100.40 & 100.40 \\
\hline USR7 & ADSL & 12.46 & 13.02 \\
\hline USR8 & ADSL2+ & 22.47 & 25.33 \\
\hline USR9 & ADSL2+ & 22.47 & 28.6 \\
\hline USR10 & ADSL2+ & 22.4 & 28.62 \\
\hline
\end{tabular}

\section{B. Downstream transmission with multiples services and DSM}

To obtain a more realistic picture of DSM performance gains, the ten-user scenario from previous section was simulated with DSM algorithms.

The different distances between transmitters and receivers leads to the generation of strong interference from the Remote Terminal lines on the lines directly connected to the Central Office. DSM algorithms (IWF and SCALE) were applied in all lines in order to minimize this effect.

Table III and Table IV show the DSM performance when using standard and measured channel models, respectively. As it can be seen, the use of DSM allowed an increase of the bit rate in almost all lines. Due the lower level of crosstalk, the results of measured channel reached better performance. Compared with IWF, SCALE achieved higher rates for both measured and standard channel models. The values between parenthesis represents the gain in percentage compared with the current SSM.

TABLE III

RATE CAPACITY IMPROVEMENTS MADE BY DSM PROCESSING USING STANDARD CHANNEL MODELS (IN MBPS).

\begin{tabular}{|c||c|c|c|}
\hline Lines & Service & IWF & \multicolumn{2}{|c|}{ SCALE } \\
\hline \hline USR1 & ADSL & $10.68(+5.12 \%)$ & $11.12(+9.45 \%)$ \\
\hline USR2 & ADSL & $10.68(+5.12 \%)$ & $11.12(+9.45 \%)$ \\
\hline USR3 & ADSL2+ & $16.26(-0.91 \%)$ & $18.2(+10.91 \%)$ \\
\hline USR 4 & ADSL2+ & $16.26(-0.91 \%)$ & $18.2(+10.91 \%)$ \\
\hline USR5 & ADSL2+ & $16.26(-0.91 \%)$ & $18.2(+10.91 \%)$ \\
\hline USR6 & VDSL2 & $115.52(+15 \%)$ & $117.24(+16.77 \%)$ \\
\hline USR7 & ADSL & $13.36(+7.2 \%)$ & $12.61(+1.2 \%)$ \\
\hline USR8 & ADSL2 & 22.51 & 22.48 \\
\hline USR9 & ADSL2+ & 22.51 & 22.48 \\
\hline USR10 & ADSL2+ & 22.51 & 22.48 \\
\hline
\end{tabular}

TABLE IV

RATE CAPACITY IMPROVEMENTS MADE BY DSM PROCESSING USING MEASURED CHANNEL (IN MBPS).

\begin{tabular}{|c||c|c|cc|}
\hline Lines & Service & \multicolumn{1}{|c|}{ IWF } & \multicolumn{2}{|c|}{ SCALE } \\
\hline \hline USR1 & ADSL & $13.39(+6.1 \%)$ & $13.41(+6.26 \%)$ \\
\hline USR2 & ADSL & $14.31(+3.5 \%)$ & $14.38(+3.98 \%)$ \\
\hline USR3 & ADSL2+ & $24.54(+1.5 \%)$ & $25.03(+3.5 \%)$ \\
\hline USR 4 & ADSL2+ & 24.79 & $25.05(+0.72 \%)$ \\
\hline USR5 & ADSL2+ & $25.07(-1.8 \%)$ & $26.02(+1.96 \%)$ \\
\hline USR6 & VDSL2 & $115.51(+15 \%)$ & $115.06(+14.06 \%)$ \\
\hline USR7 & ADSL & $14.15(+8.7 \%)$ & $14.06(+8.0 \%)$ \\
\hline USR8 & ADSL2+ & $25.46(+0.5 \%)$ & $26.17(+3.32) \%$ \\
\hline USR9 & ADSL2+ & $28.16(-1.5 \%)$ & $27.54(-3.71 \%)$ \\
\hline USR10 & ADSL2+ & 28.58 & 28.71 \\
\hline
\end{tabular}

C. Downstream Transmission with VDSL2 in a 2-User Scenario

A two-user mixed scenario has been also selected to make the comparison of achievable rate between the DSM algorithms when using values of measured and standard channel models. The scenario is depicted in Fig. 7 and consists of two VDSL2 modems in a mixed CO/RT. A variety of different target rates were set on the RT user, while the $\mathrm{CO}$ line attempted to maximize its own data rate. A maximum transmit power of $21.05 \mathrm{dBm}$ was allowed to each modem, and also the frequency band plan of up $12 \mathrm{MHz}$, as established in [19].

For comparison, a rate region for flat $\mathrm{PBO}$ have been included. In flat PBO each modem transmits the minimum possible flat PSD required to support its target rate [2]. A standard defined spectral mask was applied only to the flat power back-off (PBO) case. Note for measured and calculated channels, the rate region coincidence for ISB and SCALE where both algorithms reached the better performance.

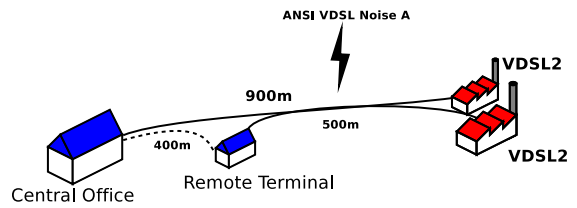

Fig. 7. 2 non-equal length users using VDSL2 service.

Fig. 8 and Fig. 9 illustrate the rate regions for measured and standard channel model corresponding to the various spectrum management algorithms. For the case of measured channel depicted in Fig. 9, the IWF reached gains near the ISB and SCALE. This is due to the lower level of crosstalk coupling, which contributes for a better performance and a transmission closer to the ideal conditions.

\section{CONCLusions}

The results highlighted the fact that FEXT coupling functions adopted in the standards are conservatives, and consequently, they influence the actual DSM algorithms performance.

To the better of the authors' knowledge, there is no published results with FEXT coupling measurements for nonequal length scenarios. On the other hand, these near-far 


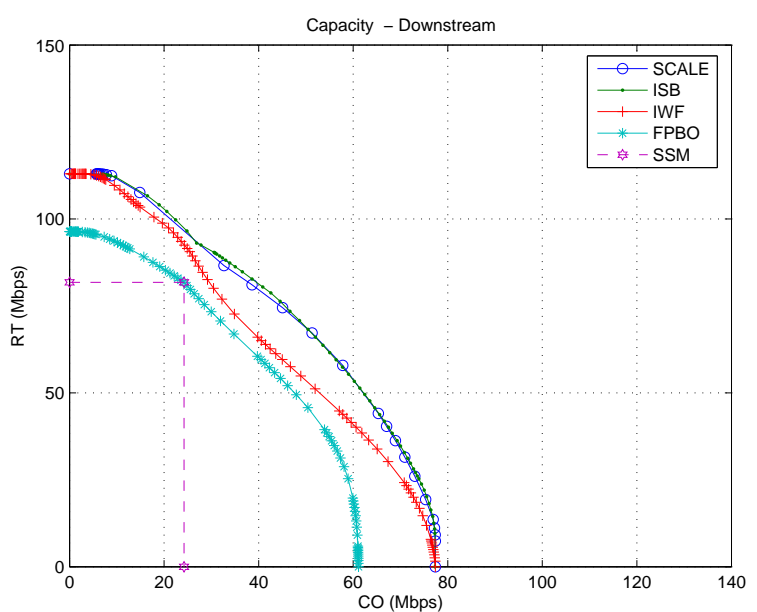

Fig. 8. Rate regions obtained for various DSM algorithms with standard channel model for 2-Users Scenario.

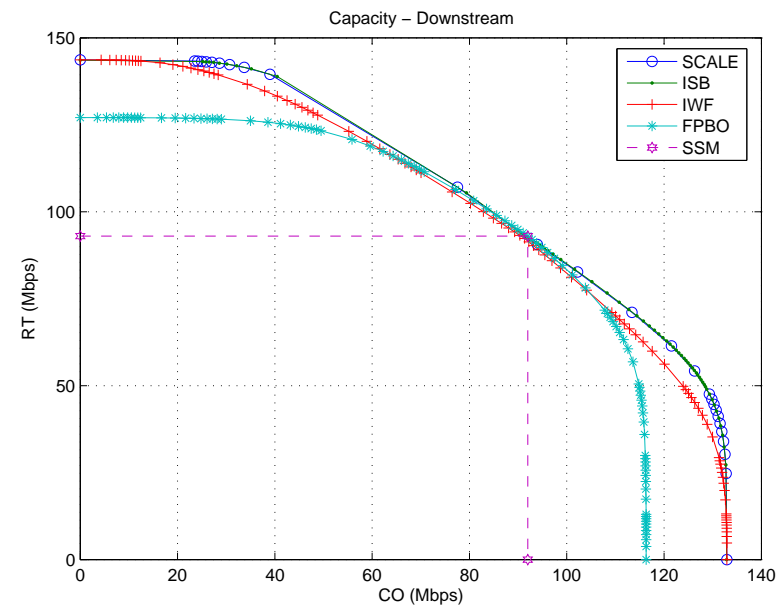

Fig. 9. Rate regions obtained for various DSM algorithms with measured channel for 2-Users Scenario.

scenarios are of special interest for DSM applications and consequently, were the focus of the the measurements and simulations.

Some open issues to be considered in next works regarding DSM performance evaluation: simulations assuming an asynchronous system (so far, only synchronous systems have been considered) [20] and deeper analysis about multiservice performance of DSM enabled systems (emphasizing VDSL2 service) for short and long measured channels (between $200 \mathrm{~m}$ and $1500 \mathrm{~m}$ ).

\section{ACKNOWLEDGMENT}

Authors would like to thank the Research and Development Center, Ericsson Telecommunications S.A., Brazil. Some of the authors want to acknowledge the financial support received from the European Commission IST 6th Framework and from the Swedish Agency for Innovation Systems, VINNOVA, through the IST - MUSE and the Eureka - Celtic BANITS projects respectively, which partially enabled this work.

\section{REFERENCES}

[1] T. Starr, J. M. Cioffi, and P. J. Silverman, Understanding Digital Subscriber Line Technology. Prentice-Hall, 1999.

[2] R. Cendrillon, M. Moonen, J. Verliden, and T. B. W. Yu, "Optimal Multi-user Spectrum Management for Digital Subscriber Lines," IEEE International Conference on Communications (ICC), vol. 1, pp. 1-5, 2004.

[3] R. Cendrillon and M. Moonen, "Iterative spectrum balancing for digital water-filling implemented on ADSL modems," in Proc. IEEE Int. Conf. on Acoust., Speech and Sig. Processing (ICASSP), May 2005.

[4] J. Papandriopoulos and J. S. Evans, "Low-Complexity Distributed Algorithms for Spectrum Balancing in Multi-User DSL Networks," 2005. [Online]. Available: http://www.cubinlab.ee.mu.oz.au/ jpap/

[5] S. G, P. D. P., and B. S.;, "Asynchronous iterative water-filling for gaussian frequency-selective interference channels: A unified framework," in SPAWC 2006, 2006.

[6] ANSI, "T1.417 - Spectrum Management fo Loop Transmission Systems, Issue 2," 2003.

[7] _ " "T1.413 - Network to customer installation interface - Asymmetric Digital Subscriber Lines (ADSL) Metallic Interface," 2004.

[8] J. Bezerra, A. C. Gomes, B. Dortschy, A. Klautau, and E. Pelaes, "The influence of dsm on legacy xDSL services," in Proc. I2TS'2006 - 5th International Information and Telecommunication Technologies Symposium, 2006.

[9] I. Conexant Systems, "Proposal for construction of a MIMO channel model for evaluation of FEXT cancellation systems - nipp-nai-2006$169, " 2006$.

[10] AT\&T, Adtran, and C. Systems, "Proposal for construction of a MIMO channel model for evaluation of FEXT cancellation systems-nipp-nai2007-008," 2007.

[11] C. Valenti, "NEXT and FEXT Models for Twisted-Pair North American Loop Plant," IEEE J. Select. Areas Commun., vol. 20, pp. 893-900, 2002.

[12] S. Galli and K. Kerpez, "Methods of summing crosstalk from mixed sources - part i: Theorectical analysis," IEEE Transations on Communication, vol. 50, pp. 453-461, march 2002.

[13] K. Kerpez and S. Galli, "Methods of summing crosstalk from mixed sources - part ii: Performance results," IEEE Transations on Communication, vol. 50, pp. 600-607, april 2002.

[14] W. Yu, G. Ginis, and J. M. Cioffi, "Distributed Power Control for Digital Subscriber Lines," IEEE J. Select. Areas Commun., vol. 20, no. 5, pp. 1105-15, June 2002.

[15] R. Cendrillon, "Multi-user signal and spectra co-ordination for digital subscriber lines," Ph.D. dissertation, Katholieke Universiteit Leuven, Dec. 2004.

[16] E. V. den Bogaert, T. Bostoen, J. V. Elsen, R. Cendrillon, and M. Moonen, "DSM in practice: Iterative water-filling implemented on ADSL modems," in Proc. IEEE Int. Conf. on Acoust., Speech and Sig. Processing (ICASSP), 2004

[17] J. Verlinden, T. Bostoen, and G. Ysebaert, "Dynamic spectrum management for digital subscriber lines - edition 2," June 2005.

[18] W. Yu and R. Lui, "Dual Methods for Nonconvex Spectrum Optimization of Multicarrier Systems," IEEE Transactions On Communications, vol. 54, pp. 1310-1322, 2006.

[19] I. S. G.993.2, "Very high speed digital subscriber line 2 (vds12)," 2006.

[20] J. Huang, R. Cendrillon, M. Chiang, and M. Moonen, "Autonomous spectrum balancing (ASB) for digital subscriber lines," in IEEE Trans. Signal Processing (Accepted), Dec. 2006. 